\title{
Dissection of the glycosylation in the biosynthesis of the heptadecaglycoside antibiotic saccharomicin $A$
}

Junfeng Zhao, Tianlu Mo, Xinhui Li, Wei Ding, and Qi Zhang*

\section{Table of Contents}

Figure S1. Schematic representation of the in-frame deletions of sam11 in S. espanaensis. $\quad$ S2

Figure S2. Schematic representation of the in-frame deletions of sam12 in S. espanaensis. $\quad$ S2

Figure S3. Schematic representation of the in-frame deletions of sam13 in S. espanaensis. $\quad$ S3

Figure S4. Schematic representation of the in-frame deletions of sam14 in S. espanaensis. $\quad$ S3

Figure S5. Schematic representation of the in-frame deletions of sam 14 in S. espanaensis. $\quad$ S4

Figure S6. Schematic representation of the in-frame deletions of sam14 in S. espanaensis. $\quad$ S4

Figure S7. Schematic representation of the in-frame deletions of sam14 in S. espanaensis. S5

Figure S8. Schematic representation of the in-frame deletions of sam14 in S. espanaensis. S5

Figure S9. Schematic representation of the in-frame deletions of sam 14 in S. espanaensis. $\quad$ S6

Figure S10. Schematic representation of the in-frame deletions of sam 14 in S. espanaensis. S6

$\begin{array}{ll}\text { Figure S11. HR-MS/MS characterization of SA-A. } & \text { S7 }\end{array}$

$\begin{array}{lr}\text { Figure S12. HR-MS/MS characterization of M13. } & \text { S7 }\end{array}$

$\begin{array}{lr}\text { Figure S13. HR-MS/MS characterization of M12. S8 } & \text { S12 }\end{array}$

$\begin{array}{ll}\text { Figure S14. HR-MS/MS characterization of M11-1. } & \text { S8 }\end{array}$

Figure S15. HR-MS/MS characterization of M11-2.

$\begin{array}{lr}\text { Figure S16. HR-MS/MS characterization of M14. S9 } & \text { S9 }\end{array}$

$\begin{array}{lr}\text { Figure S17. HR-MS/MS characterization of M15. } & \text { S10 }\end{array}$

$\begin{array}{lr}\text { Figure S18. HR-MS/MS characterization of M16-1. } & \text { S10 }\end{array}$

$\begin{array}{ll}\text { Figure S19. HR-MS/MS characterization of M16-2. } & \text { S11 }\end{array}$

$\begin{array}{ll}\text { Figure S20. HR-MS/MS characterization of M18. } & \text { S11 }\end{array}$

$\begin{array}{lr}\text { Figure S21. HR-MS/MS characterization of M17. } & \text { S12 }\end{array}$

$\begin{array}{ll}\text { Figure S22. Bayesian MCMC phylogeny of Sam11-homologous proteins. } & \text { S12 }\end{array}$

Table S1. The fermentation titers for SA-A and the structural analogs. S13

$\begin{array}{ll}\text { Table S2. PCR primers used in this study. } & \text { S14 }\end{array}$

$\begin{array}{ll}\text { Table S3. Strains used in this study. } & \text { S16 }\end{array}$

$\begin{array}{lr}\text { Table S4. Plasmids used in this study. } & \text { S17 }\end{array}$

$\begin{array}{ll}\text { References } & \text { S18 }\end{array}$ 
Figure S1. Schematic representation of the in-frame deletions of sam11 in S. espanaensis. The probe for southern blot is shown as a purple bar. The arrows indicate the expected size of the fragments from the wild-type and mutant in the southern blot analysis, respectively.

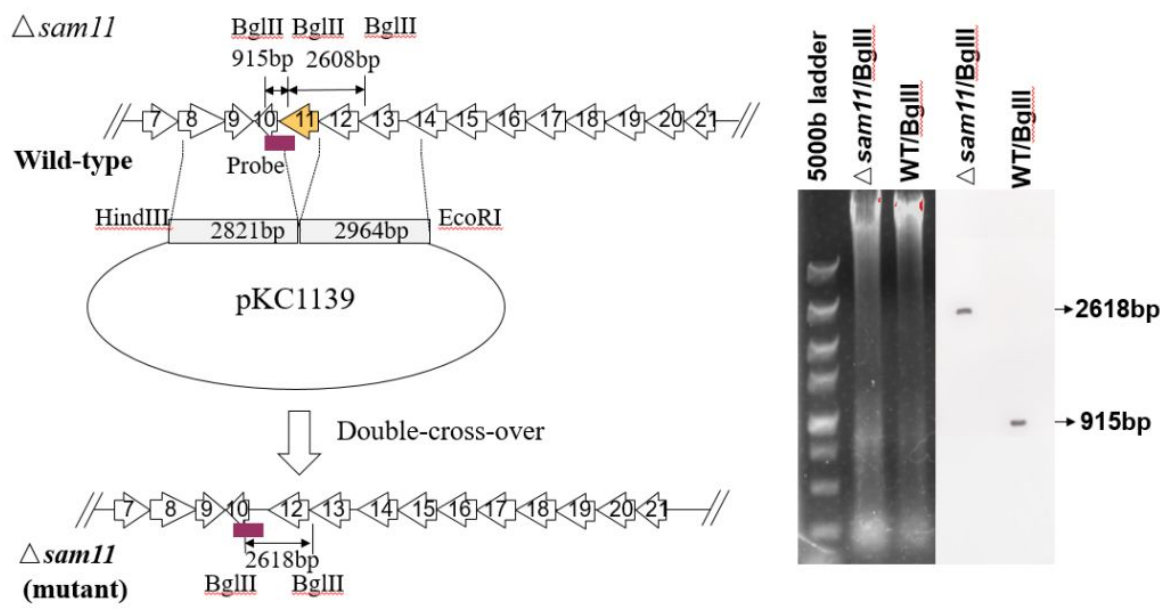

Figure S2. Schematic representation of the in-frame deletions of sam12 in S. espanaensis. The probe for southern blot is shown as a purple bar. The arrows indicate the expected size of the DNA fragments from the wild-type and mutant in the southern blot analysis, respectively.

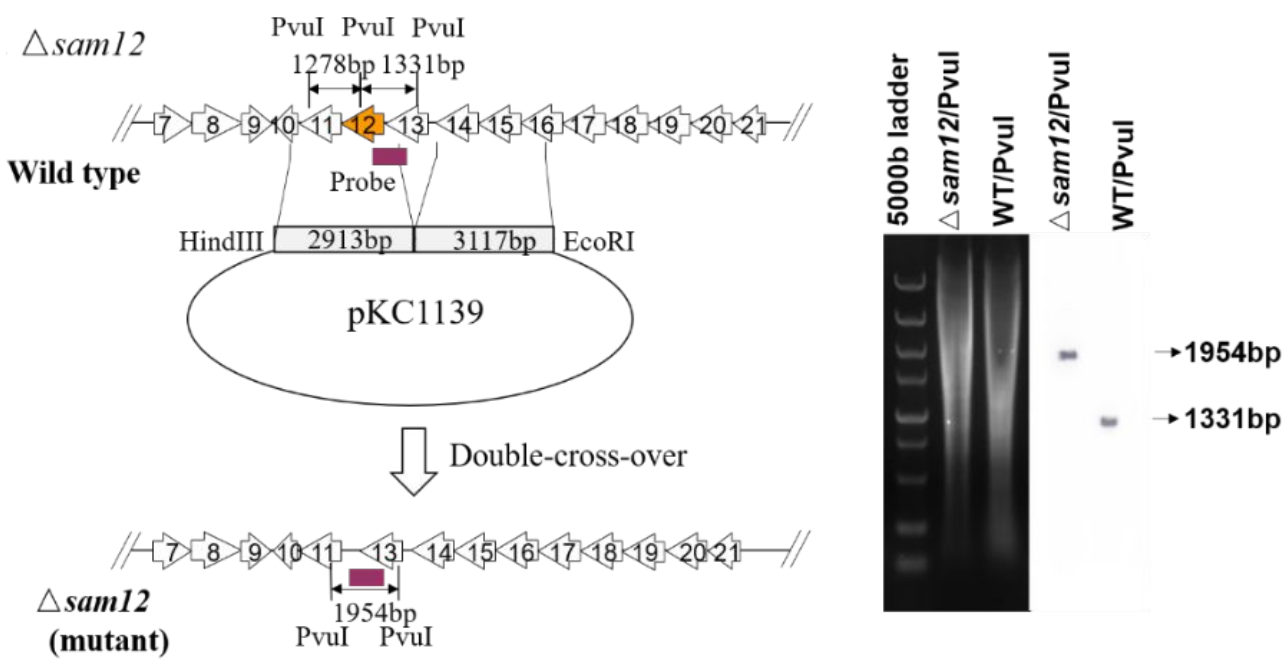


Figure S3. Schematic representation of the in-frame deletions of sam13 in S. espanaensis. The probe for southern blot is shown as a purple bar. The arrows indicate the expected size of the DNA fragments from the wild-type and mutant in the southern blot analysis, respectively.

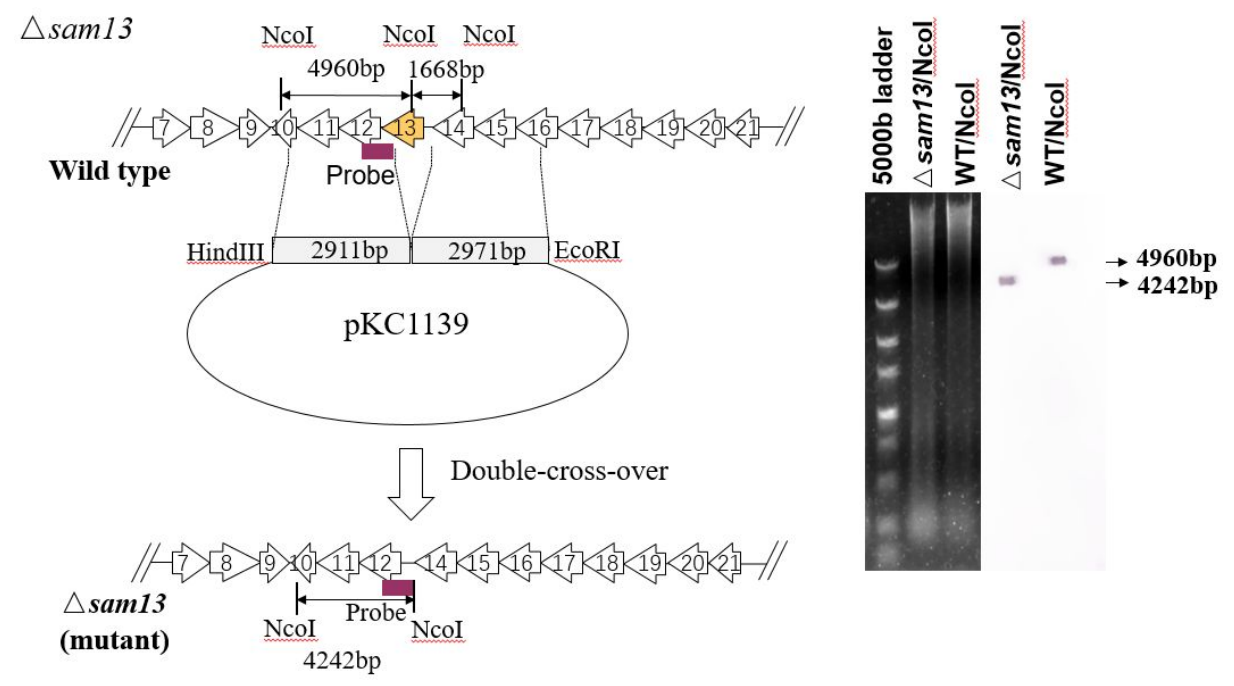

Figure S4. Schematic representation of the in-frame deletions of sam14 in S. espanaensis. The probe for southern blot is shown as a purple bar. The arrows indicate the expected size of the DNA fragments from the wild-type and mutant in the southern blot analysis, respectively.

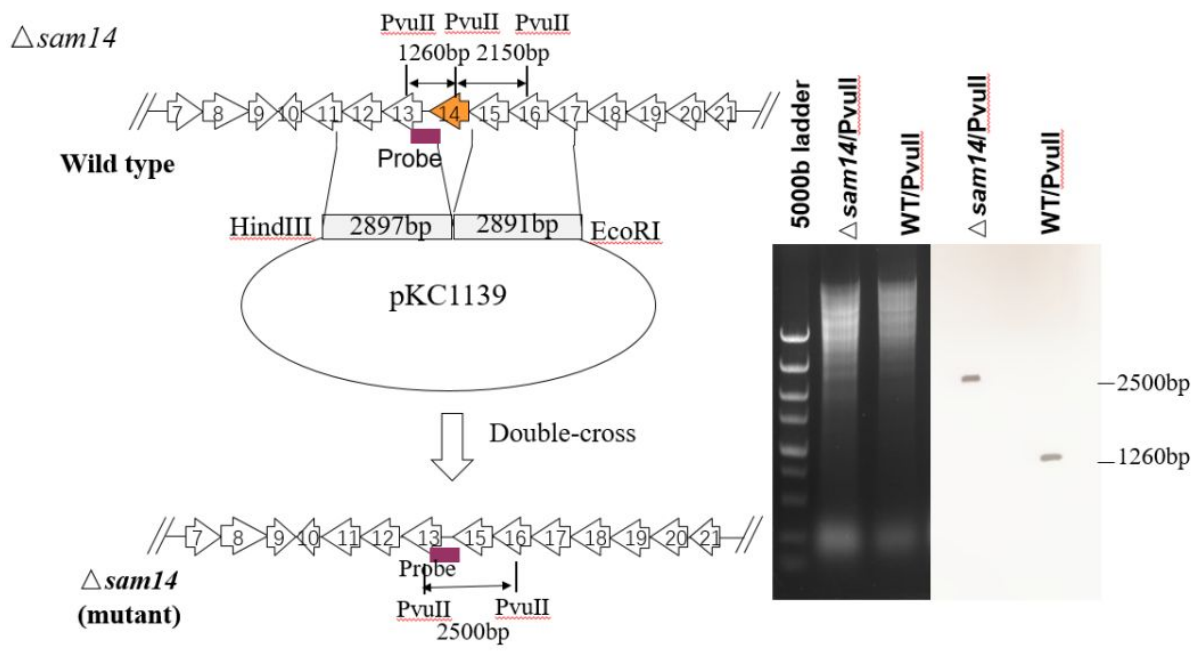


Figure S5. Schematic representation of the in-frame deletions of sam15 in S. espanaensis. The probe for southern blot is shown as a purple bar. The arrows indicate the expected size of the DNA fragments from the wild-type and mutant in the southern blot analysis, respectively.

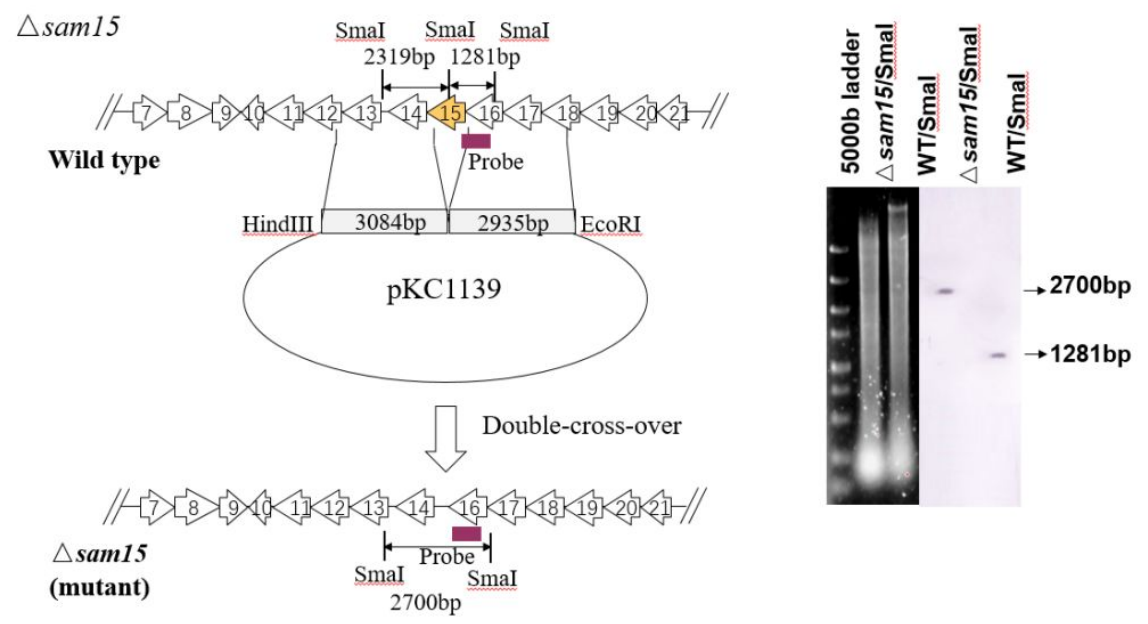

Figure S6. Schematic representation of the in-frame deletions of sam16 in S. espanaensis. The probe for southern blot is shown as a purple bar. The arrows indicate the expected size of the DNA fragments from the wild-type and mutant in the southern blot analysis, respectively.

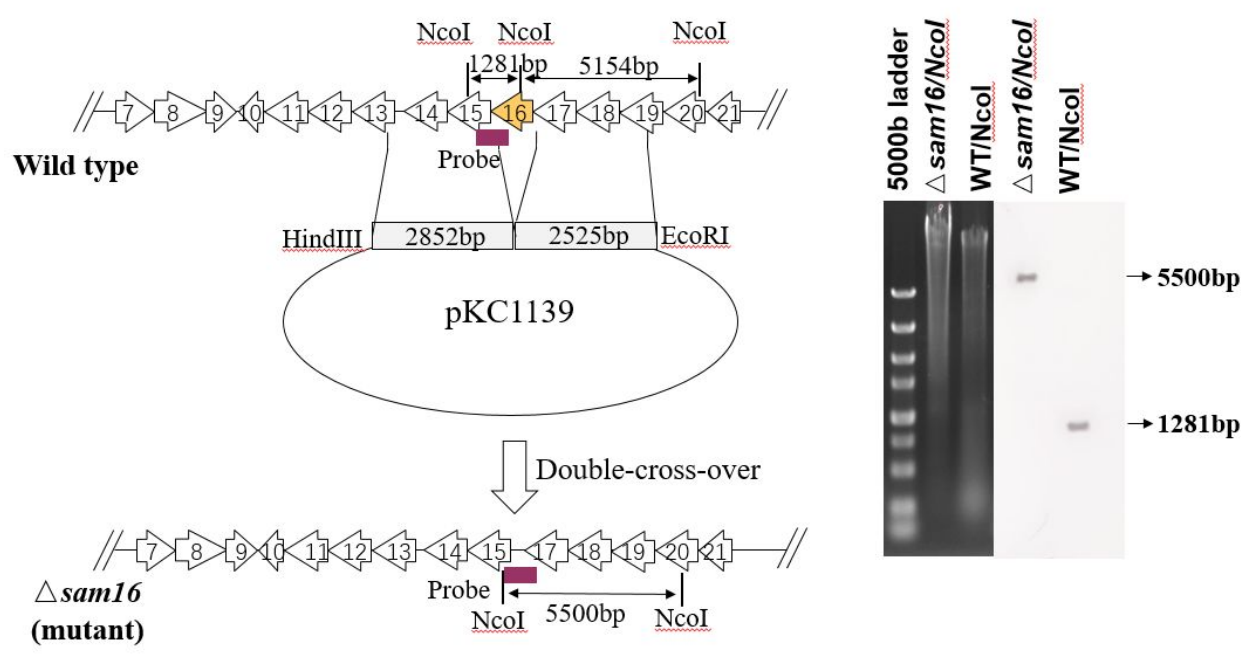


Figure S7. Schematic representation of the in-frame deletions of sam17 in S. espanaensis. The probe for southern blot is shown as a purple bar. The arrows indicate the expected size of the DNA fragments from the wild-type and mutant in the southern blot analysis, respectively.

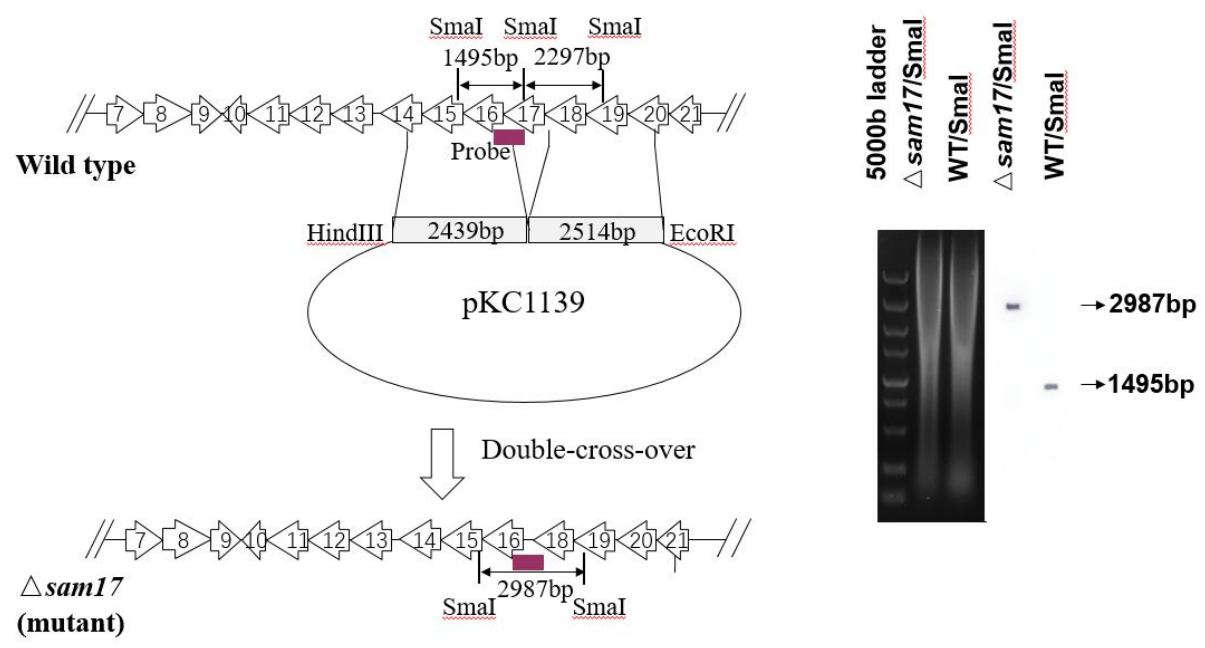

Figure S8. Schematic representation of the in-frame deletions of sam 18 in S. espanaensis. The probe for southern blot is shown as a purple bar. The arrows indicate the expected size of the DNA fragments from the wild-type and mutant in the southern blot analysis, respectively.

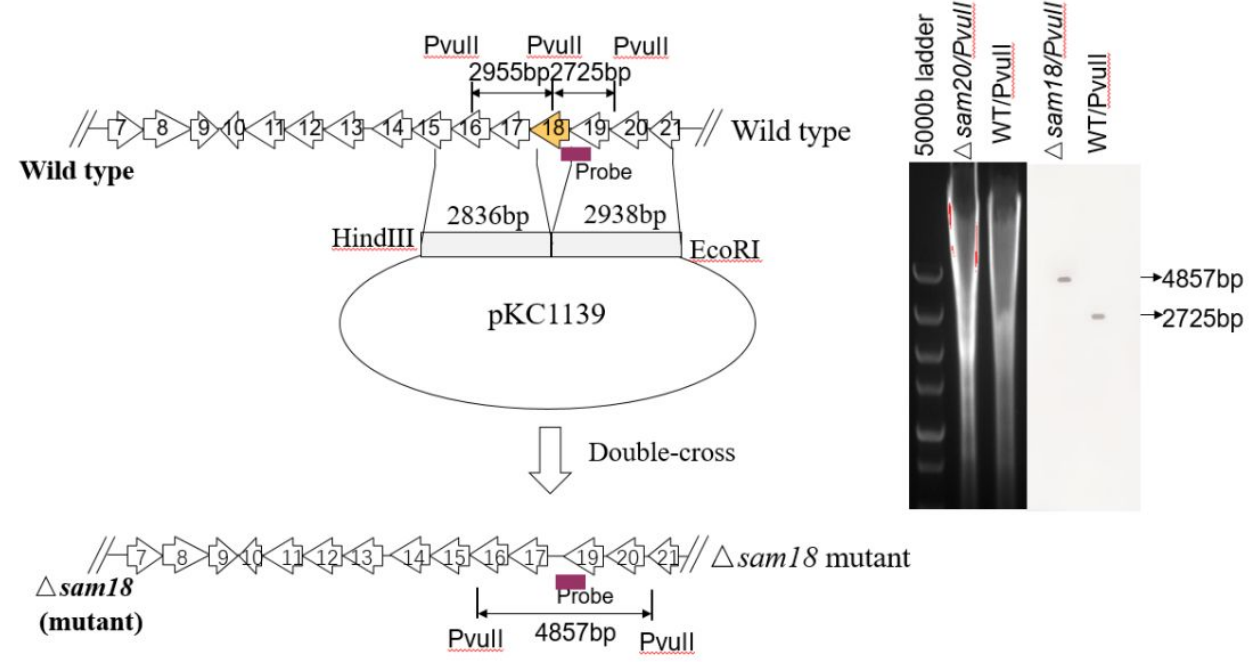


Figure S9. Schematic representation of the in-frame deletions of sam19 in S. espanaensis. The probe for southern blot is shown as a purple bar. The arrows indicate the expected size of the DNA fragments from the wild-type and mutant in the southern blot analysis, respectively.

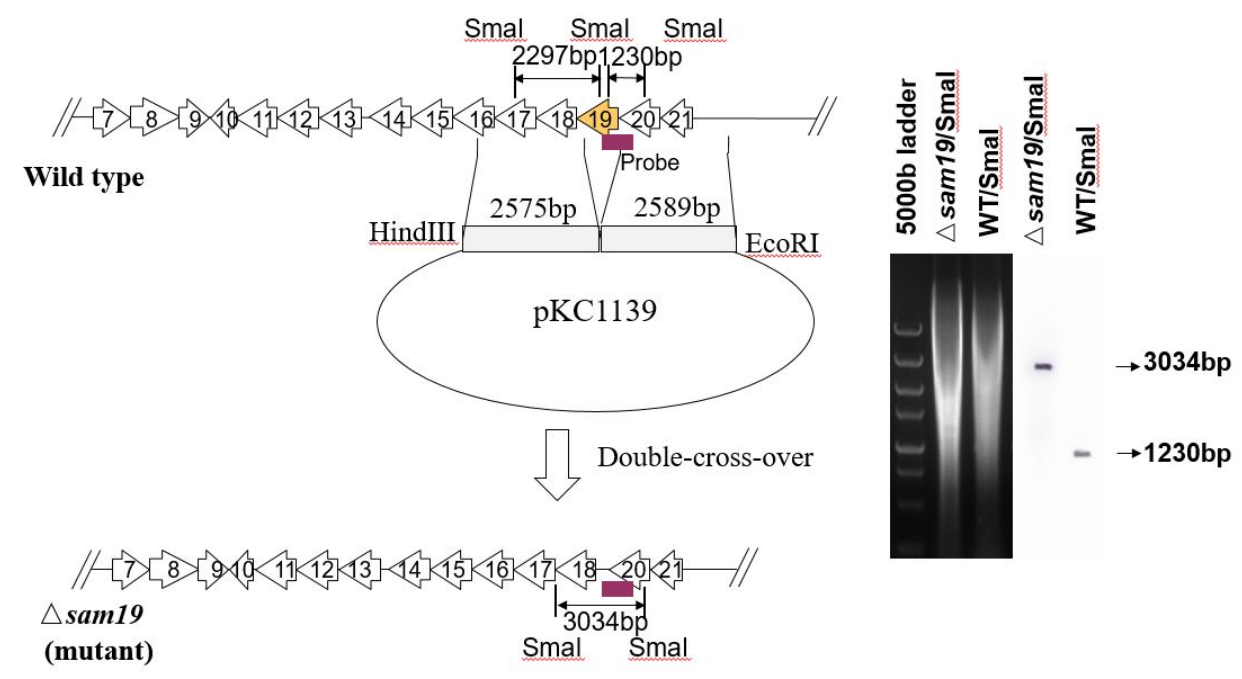

Figure S10. Schematic representation of the in-frame deletions of sam 20 in S. espanaensis. The probe for southern blot is shown as a purple bar. The arrows indicate the expected size of the DNA fragments from the wild-type and mutant in the southern blot analysis, respectively.
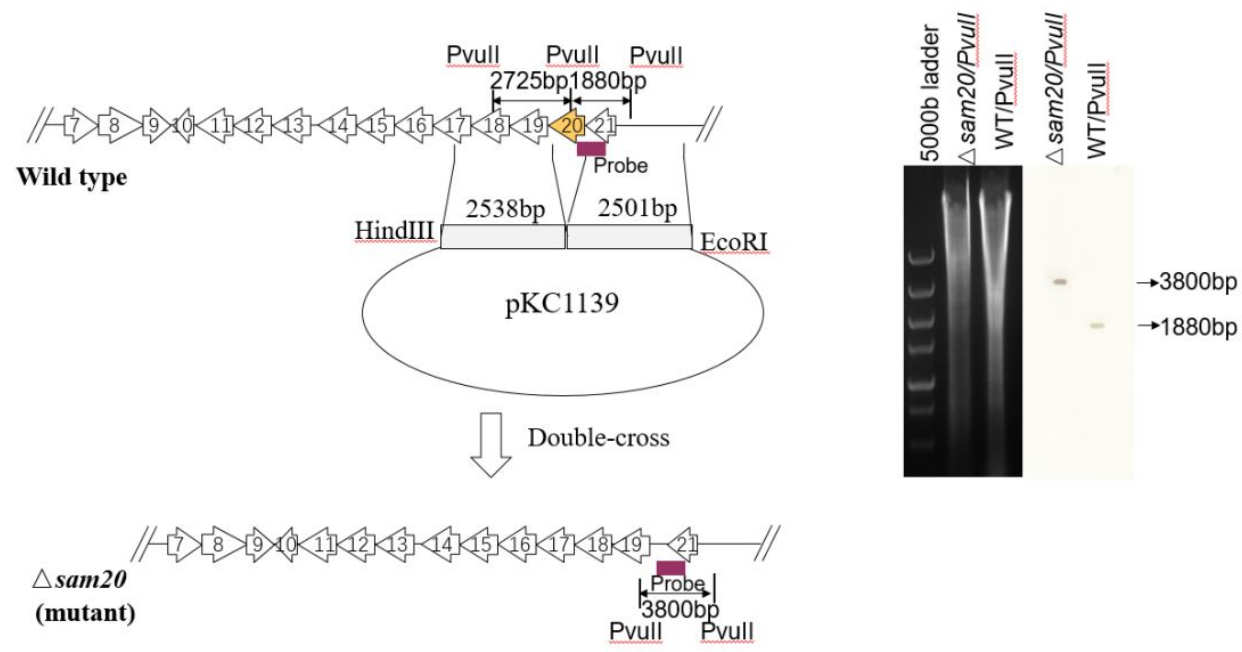
Figure S11. HR-MS/MS characterization of SA-A, showing the MS/MS spectrum and the CIDinduced fragment ions. A number with an asterisk indicates this fragment ion has a neutral loss of $\mathrm{SO}_{3}$ (sulfate in sFuc), and (+2) indicates this ion is in two-charge state.
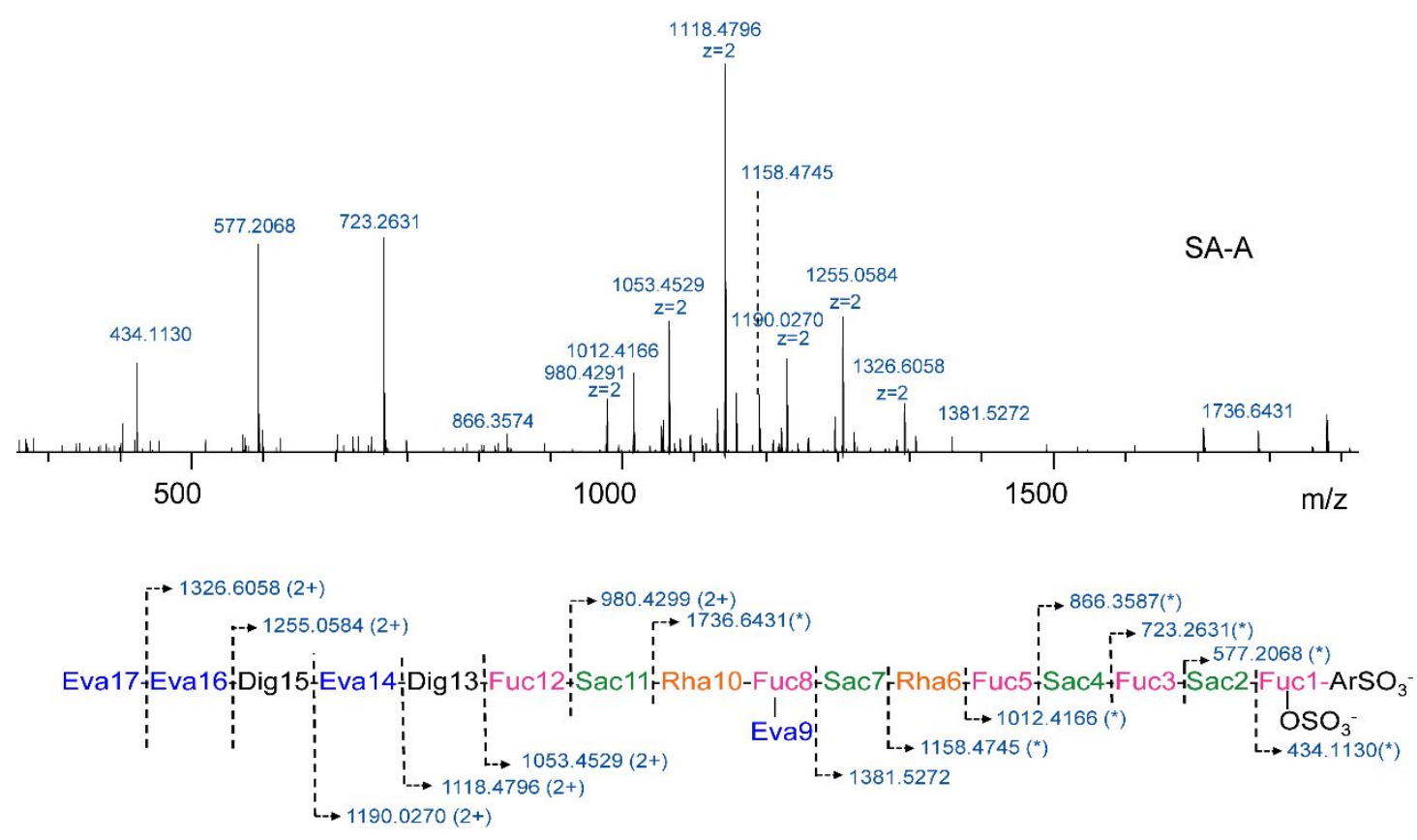

Figure S12. HR-MS/MS characterization of M13, showing the MS/MS spectrum and the CIDinduced fragment ions. A number with an asterisk indicates this fragment ion has a neutral loss of $\mathrm{SO}_{3}$ (sulfate in sFuc), and (+2) indicates this ion is in two-charge state. Red ions are those expected different from those of SA-A.
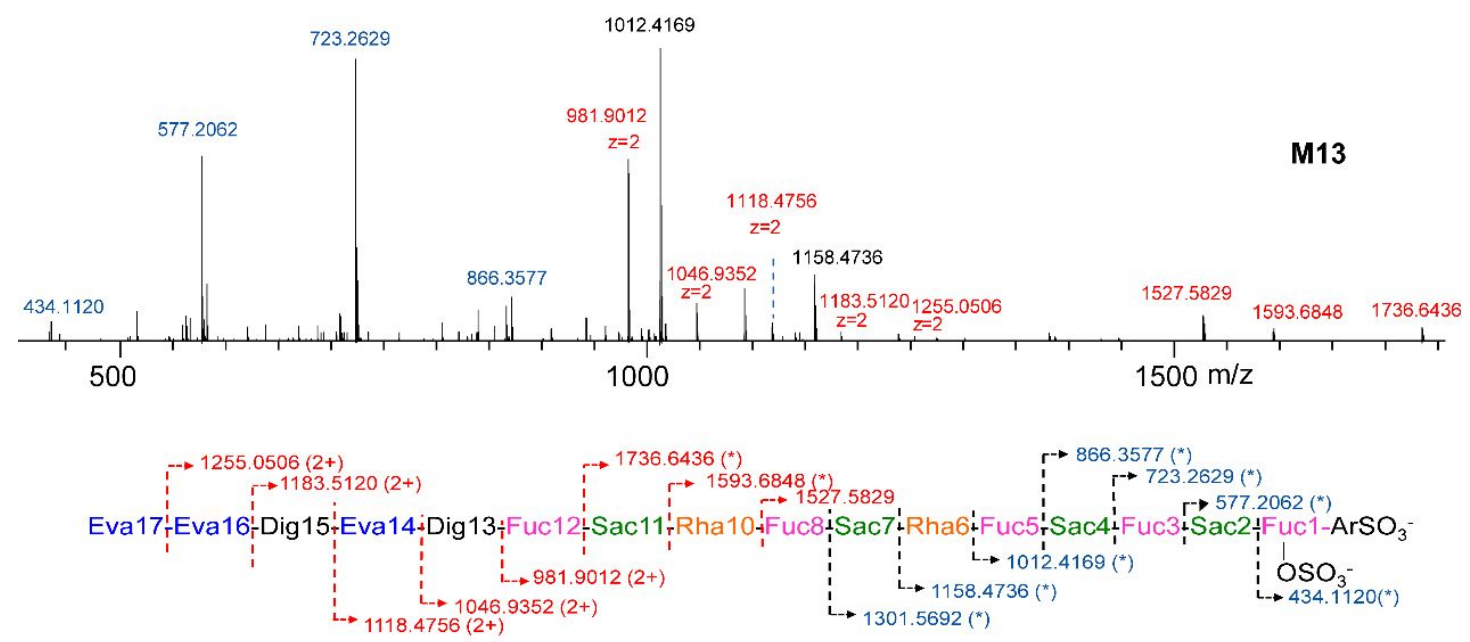
Figure S13. HR-MS/MS characterization of M12, showing the MS/MS spectrum and the CIDinduced fragment ions. $(+2)$ indicates this ion is in two-charge state.
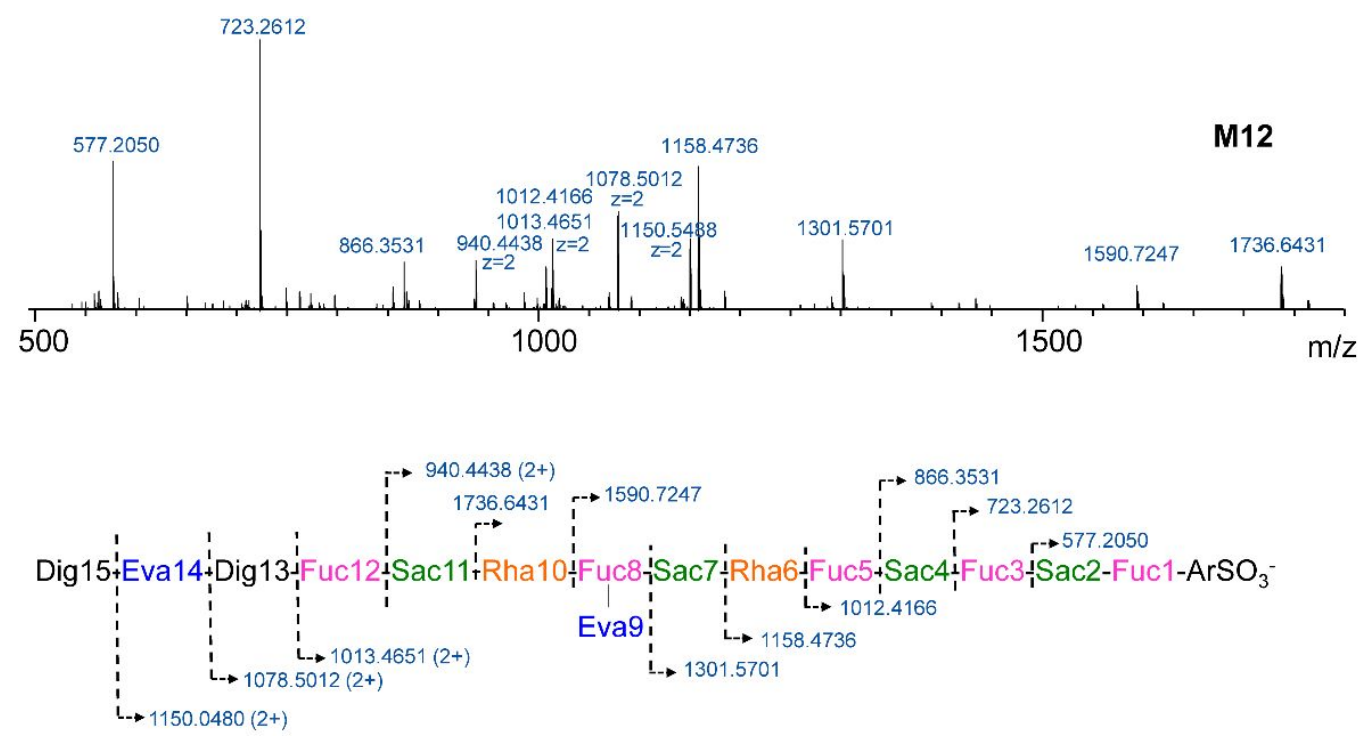

Figure S14. HR-MS/MS characterization of M11-1, showing the MS/MS spectrum and the CIDinduced fragment ions. $(+2)$ indicates this ion is in two-charge state.
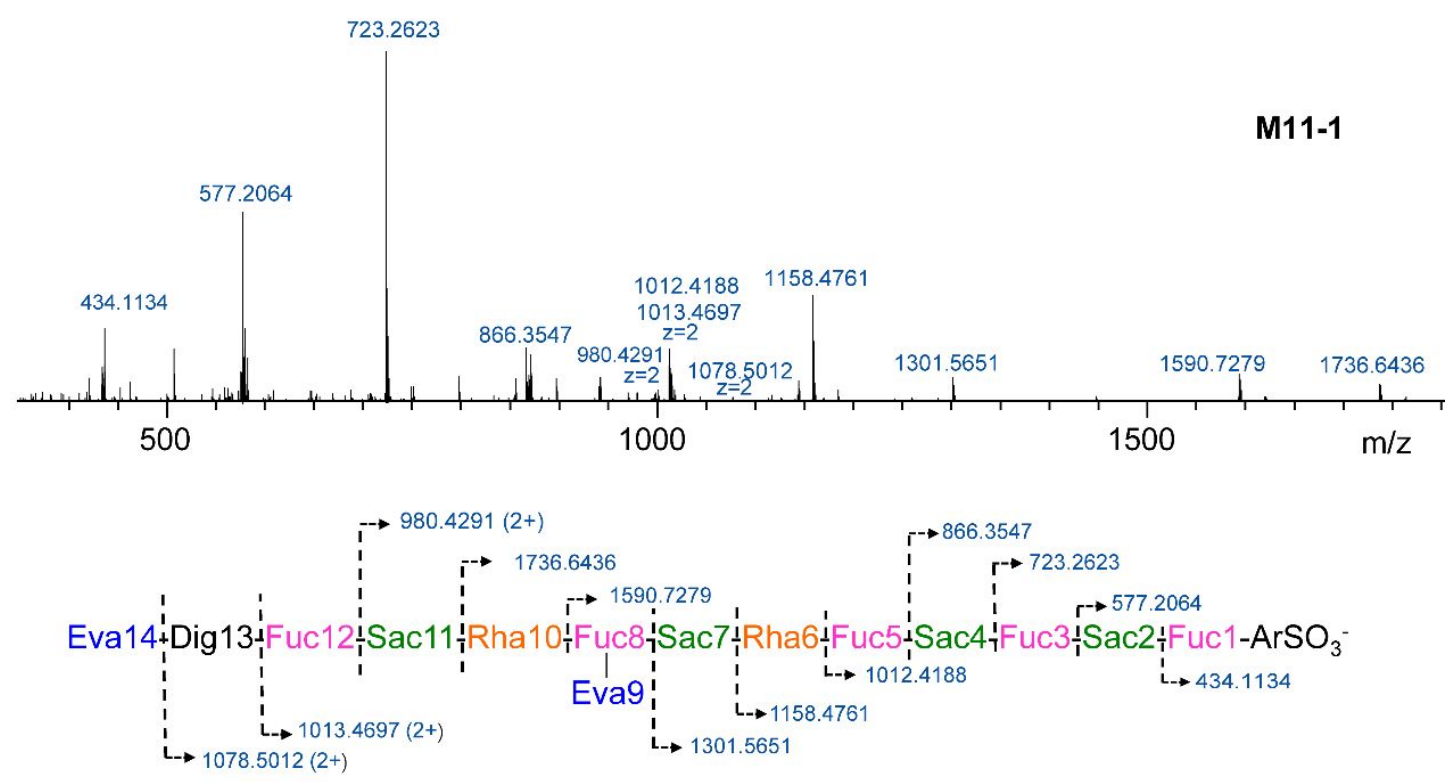
Figure S15. HR-MS/MS characterization of M11-2, showing the MS/MS spectrum and the CIDinduced fragment ions. A number with an asterisk indicates this fragment ion has a neutral loss of $\mathrm{SO}_{3}$ (sulfate in sFuc), and (+2) indicates this ion is in two-charge state. Red ions are those expected different from those of SA-A.

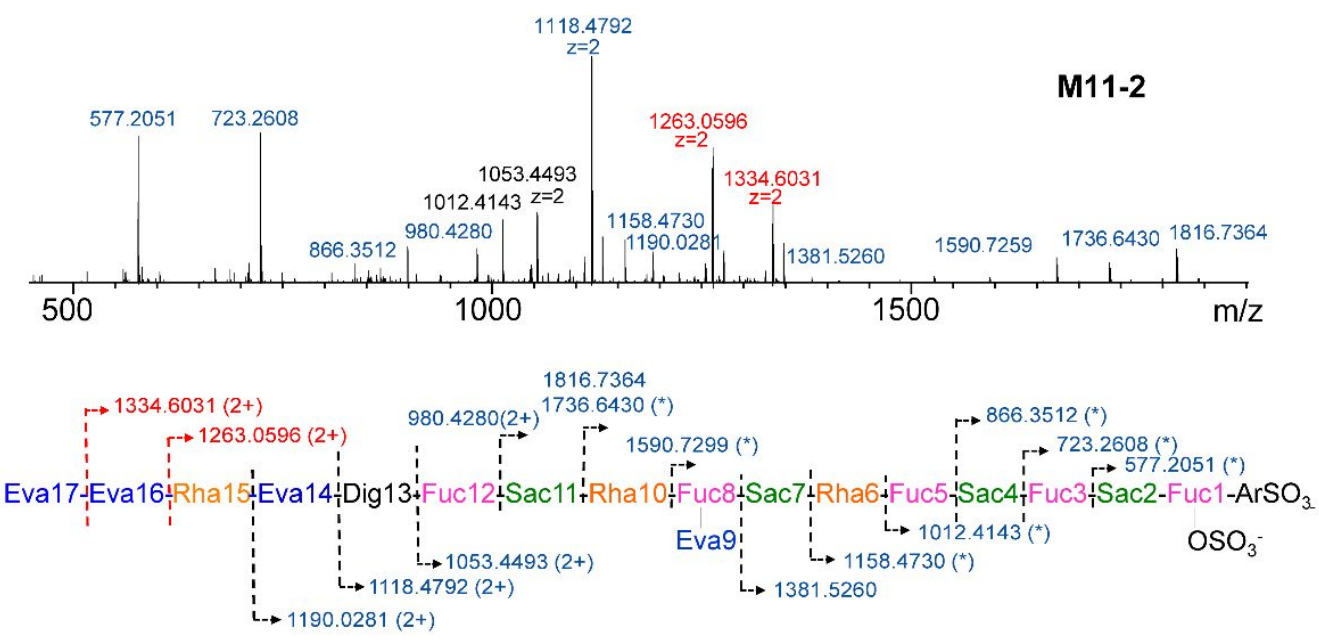

Figure S16. HR-MS/MS characterization of M14, showing the MS/MS spectrum and the CIDinduced fragment ions. $(+2)$ indicates this ion is in two-charge state.
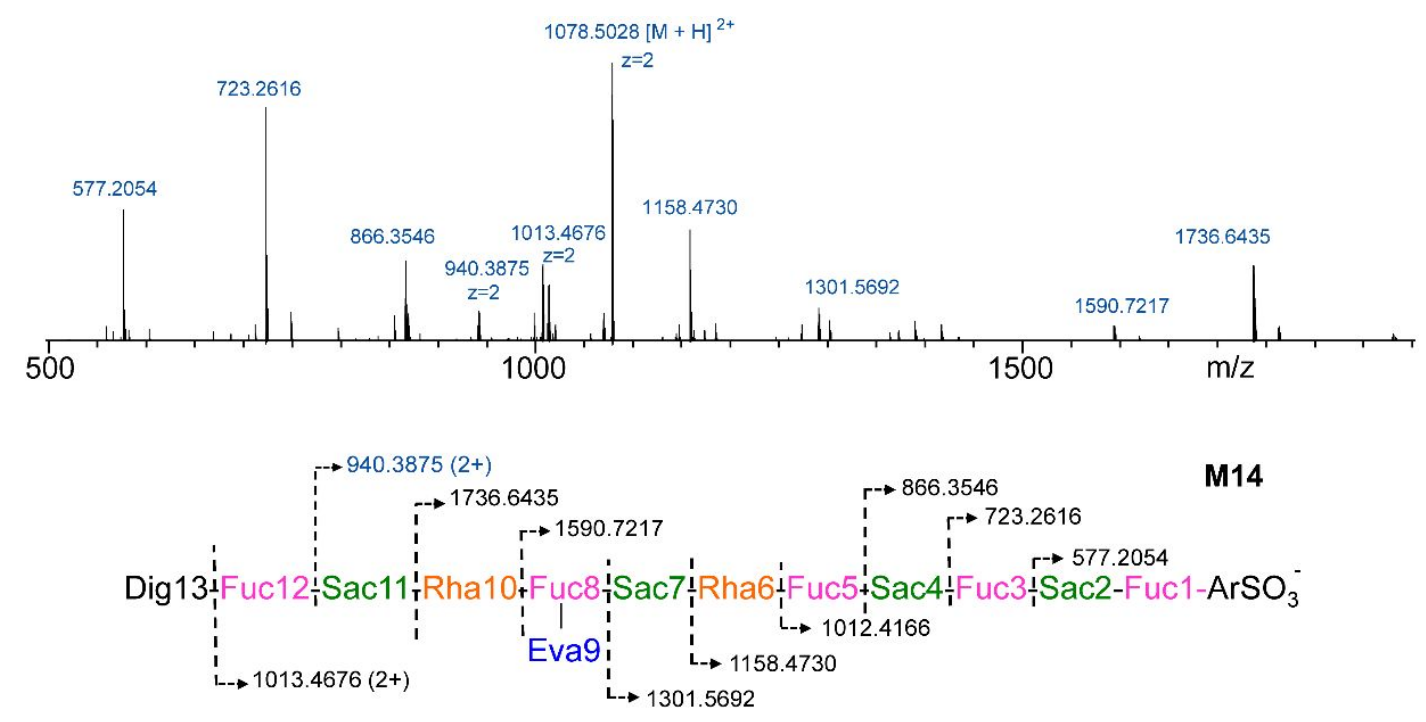
Figure S17. HR-MS/MS characterization of M15, showing the MS/MS spectrum and the CIDinduced fragment ions. A number with an asterisk indicates this fragment ion has a neutral loss of $\mathrm{SO}_{3}$ (sulfate in sFuc), and (+2) indicates this ion is in two-charge state. Red ions are those expected different from those of SA-A.
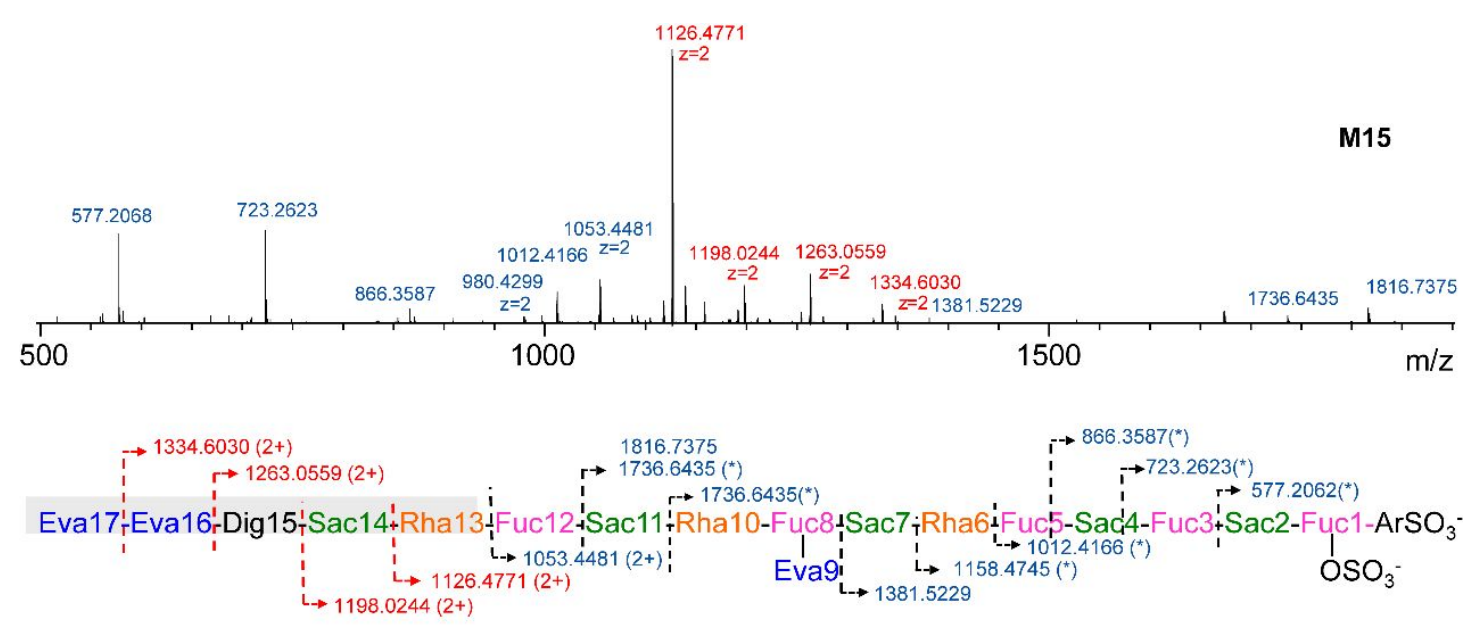

Figure S18. HR-MS/MS characterization of M16-1, showing the MS/MS spectrum and the CIDinduced fragment ions.

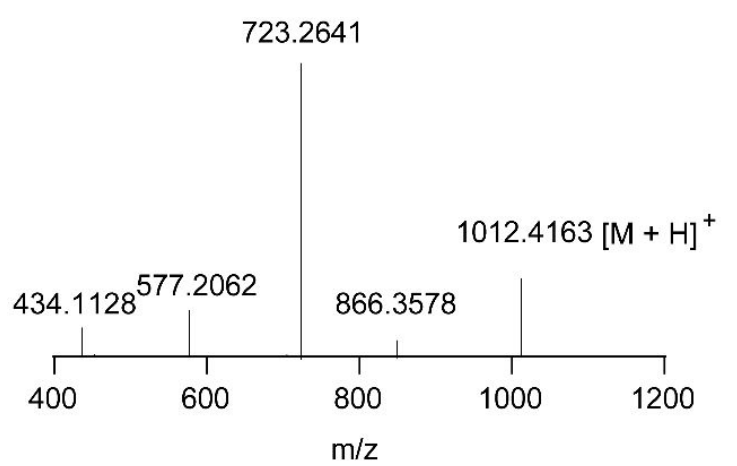

M16-1

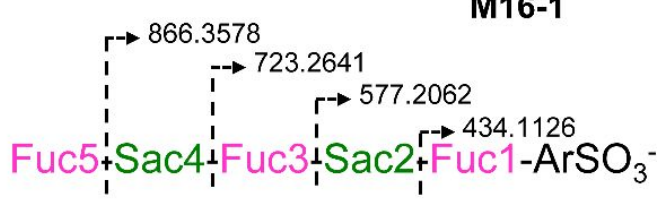


Figure S19. HR-MS/MS characterization of M16-2, showing the MS/MS spectrum and the CIDinduced fragment ions. A number with an asterisk indicates this fragment ion has a neutral loss of $\mathrm{SO}_{3}$ (sulfate in sFuc), and (+2) indicates this ion is in two-charge state. Red ions are those expected different from those of SA-A.
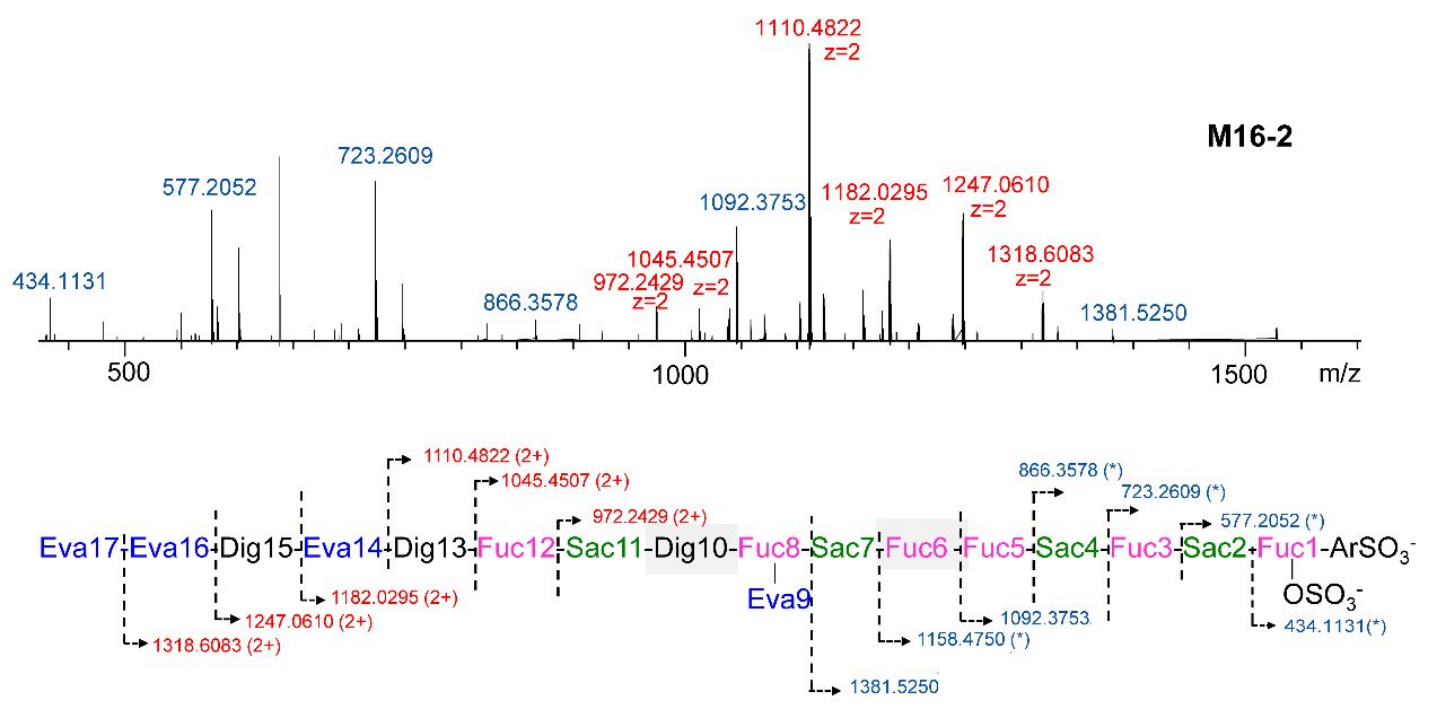

Figure S20. HR-MS/MS characterization of M18, showing the MS/MS spectrum and the CIDinduced fragment ions.

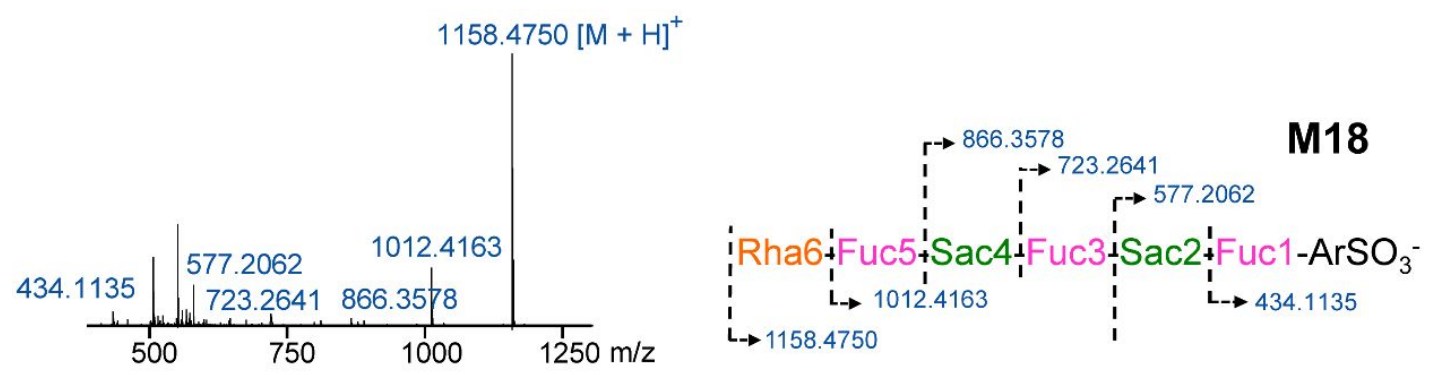


Figure S21. HR-MS/MS characterization of M17, showing the MS/MS spectrum and the CIDinduced fragment ions.
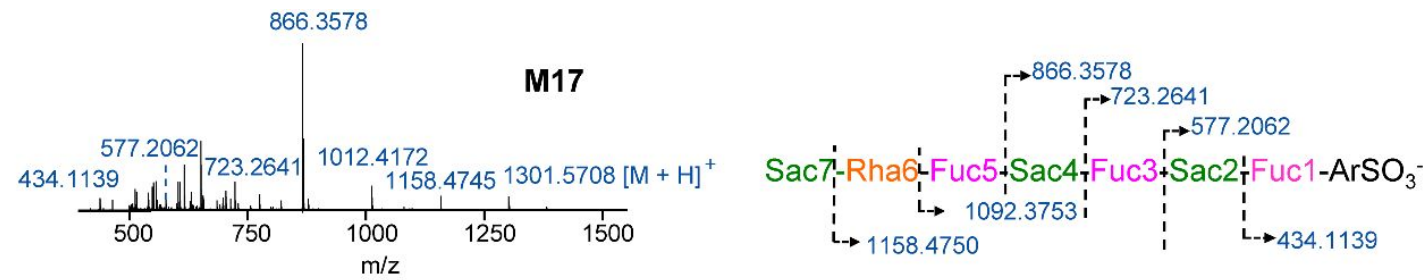

Figure S22. Bayesian MCMC phylogeny of Sam11-homologous proteins. The proteins are shown with the NCBI accession numbers and their host strains. The GTs involved in SA-A biosynthesis are shown in red. Bayesian inferences of posterior probabilities are indicated along the branches.

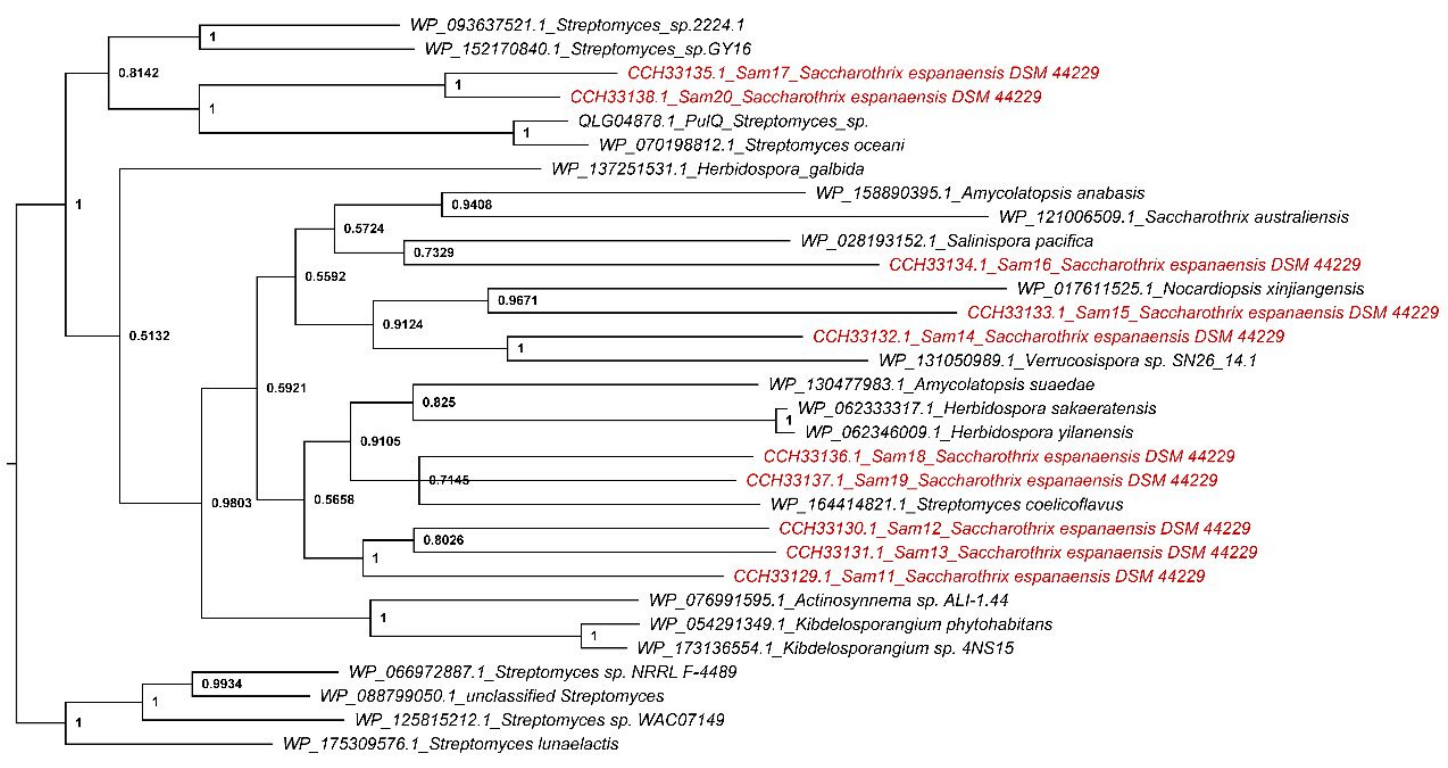


Table S1. The fermentation titers for SA-A and the structural analogs produced by the $S$. espanaensis wide type and mutant strains.

\begin{tabular}{|c|c|c|c|c|c|}
\hline Compound & Yield (mg/L) & Strain & Compound & Yield (mg/L) & Strain \\
\hline SA-A & $1.6 \pm 0.3$ & wild type & SA-B & $0.20 \pm 0.03$ & wild type \\
\hline M11-1 & $0.44 \pm 0.04$ & $\Delta s a m 11$ & M11-2 & $0.20 \pm 0.04$ & $\Delta s a m 11$ \\
\hline M12 & $0.80 \pm 0.05$ & $\Delta \operatorname{sam} 12$ & M13 & $1.4 \pm 0.3$ & $\Delta \operatorname{sam} 13$ \\
\hline M14 & $0.19 \pm 0.05$ & $\Delta \operatorname{sam} 14$ & M15 & $1.2 \pm 0.3$ & $\Delta \operatorname{sam} 13$ \\
\hline M16-1 & $0.16 \pm 0.06$ & $4 \operatorname{sam} 16$ & M16-2 & $0.08 \pm 0.02$ & $4 \operatorname{sam} 16$ \\
\hline M17 & $0.17 \pm 0.05$ & $4 \operatorname{sam} 17$ & M18 & $0.18 \pm 0.04$ & $4 \operatorname{sam} 18$ \\
\hline SA-A & $0.39 \pm 0.04$ & $\Delta \operatorname{sam} 11:: \operatorname{sam} 11$ & SA-A & $0.47 \pm 0.03$ & $\Delta \operatorname{sam} 12: \because \operatorname{sam} 12$ \\
\hline SA-A & $0.59 \pm 0.02$ & 4sam13::sam 13 & SA-A & $0.37 \pm 0.02$ & $\Delta \operatorname{sam} 14:: \operatorname{sam} 14$ \\
\hline SA-A & $0.36 \pm 0.08$ & 4sam15::sam 15 & SA-A & $0.29 \pm 0.06$ & $\Delta \operatorname{sam16}:: \operatorname{sam} 16$ \\
\hline SA-A & $0.25 \pm 0.04$ & $\Delta \operatorname{sam} 17:: \operatorname{sam} 17$ & SA-A & $0.37 \pm 0.07$ & $\Delta \operatorname{sam} 18:: \operatorname{sam} 18$ \\
\hline SA-A & $0.17 \pm 0.03$ & 4sam19::sam19 & SA-A & $0.23 \pm 0.04$ & $\Delta \operatorname{sam} 20 \because \because \operatorname{sam} 20$ \\
\hline
\end{tabular}


Table S2. PCR primers used in this study.

\begin{tabular}{|c|c|}
\hline Primer & Sequence \\
\hline sam11 Left F & CGACGGCCAGTGCCAAGCTTCTGTGCTCAACCACCGGTCCGCCCT \\
\hline sam11 Left R & TTCGAGGGGCCGCTGCTGTCGCAGCTGCTCGTCGAG \\
\hline sam11 Right $\mathrm{F}$ & GACAGCAGCGGCCCCTCGAAGGTGATCAGGTCGGTGAAC \\
\hline sam11 Right $\mathrm{R}$ & TATGACATGATTACGAATTCGTGCCGACCCCTCTCCCACTTGTGG \\
\hline HB $\operatorname{sam} 11 \mathrm{~F}$ & GCTATGACATGATTACGAATTCTCACTGCGGCGCGGGCCGGTG \\
\hline \multirow[t]{2}{*}{ HB $\operatorname{sam} 11 \mathrm{R}$} & CGGTTGGTAGGATCCACATATGATGCGCGTTTTGTTCACCGTCAACCCGGAGAA \\
\hline & GACC \\
\hline sam12 Left F & CGACGGCCAGTGCCAAGCTTCAAGGTCTCGTTCCGGCTCGCCGAC \\
\hline sam12 Left R & AGCACGTTCGCCATGCACGGCGTCCCGCAGCTCCTC \\
\hline sam12 Right $\mathrm{F}$ & ACGCCGTGCATGGCGAACGTGCTGAAGTTCTCCAGGTGGTGGCACTT \\
\hline sam12 Right $\mathrm{R}$ & TATGACATGATTACGAATTCACCGCCGGGCACGAGGTGCTCTTCGCCGGTCA \\
\hline \multirow[t]{2}{*}{ HB $\operatorname{sam} 12 \mathrm{~F}$} & GCTATGACATGATTACGAATTCTCACGCACCTGTCCGGTACTTCGCGGTCAGCTC \\
\hline & TT \\
\hline HB $\operatorname{sam} 12 \mathrm{R}$ & CGGTTGGTAGGATCCACATATGCGCGTGCTTTTCGTGGCCAGCGCGG \\
\hline sam13 Left F & 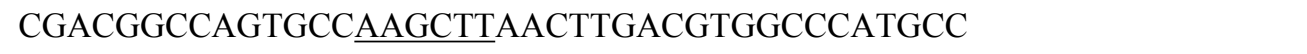 \\
\hline sam13 Left R & CTCACGCTGCCGCACCACTTCGACGAGCCGAT \\
\hline sam13 Right F & CGAAGTGGTGCGGCAGCGTGAGTTCCCAGGTGGCGTTGTCGG \\
\hline sam13 Right $\mathrm{R}$ & TATGACATGATTACGAATTCATGATGCCGATGCGCGTGCTGTTCGCCACCTA \\
\hline \multirow[t]{2}{*}{ HB $\operatorname{sam} 13 \mathrm{~F}$} & GCTATGACATGATTACGAATTCTCACCGGCCGGCCCGTACCGCGGCGGCGAGTT \\
\hline & CTTCCA \\
\hline \multirow[t]{2}{*}{ HB $\operatorname{sam} 13 \mathrm{R}$} & CGGTTGGTAGGATCCACATATGGTGCGCGTGCTATTCACCGCCAACCCGGGGAC \\
\hline & GAGC \\
\hline sam14 Left F & CGACGGCCAGTGCCAAGCTTCGCCAGTTGGAGGCTGGCCGGCA \\
\hline sam14 Left R & GACGGTCCGGCCTTCGCCGCCCT \\
\hline sam14 Right $\mathrm{F}$ & GGGCGGCGAAGGCCGGACCGTCGTGCAGGTACTCCCAGCTCGCCTTG \\
\hline sam14 Right $\mathrm{R}$ & TATGACATGATTACGAATTCGCAATCCGCTGGCACAAGACGAGCAACGTG \\
\hline HB sam $14 \mathrm{~F}$ & GCTATGACATGATTACGAATTCTCACCGGGTCCGGTGCGCGG \\
\hline HB $\operatorname{sam} 14 \mathrm{R}$ & CGGTTGGTAGGATCCACATATGCGGATCCTGTTCGTCGCCAACCCGGAGAAG \\
\hline sam15 Left F & CGACGGCCAGTGCCAAGCTTAGTTCTTCCAGCTGTGGCACCACA \\
\hline sam15 Left R & TGCTCGGTGATCATCCACCACGCCGGGT \\
\hline sam15 Right $\mathrm{F}$ & CGTGGTGGATGATCACCGAGCACAGTCCCGTGACGATCCAGTTGTGGT \\
\hline sam15 Right $\mathrm{R}$ & TATGACATGATTACGAATTCTTCGACGAGCCGGAGCTGGCCCGCCG \\
\hline HB $\operatorname{sam} 15 \mathrm{~F}$ & GCTATGACATGATTACGAATTCTCAGCCCCCGCCGACGGTGAGTTC \\
\hline
\end{tabular}




\begin{tabular}{|c|c|}
\hline HB $\operatorname{sam} 15 \mathrm{R}$ & CGGTTGGTAGGATCCACATATGATGATGCCGATGCGCGTGCTGTTCGC \\
\hline sam16 Left F & CGACGGCCAGTGCCAAGCTTTCCACAAGTGGGAGAGGGGTCGG \\
\hline sam16 Left R & CGACGGCCAGTGCCAAGCTTTCCACAAGTGGGAGAGGGGTCGG \\
\hline sam16 Right $\mathrm{F}$ & CAGCTGACCCTGCCCTGGGAC \\
\hline sam16 Right $\mathrm{R}$ & GTCCCAGGGCAGGGTCAGCTGCTGCTTGCCGCCCAGCCAGATCC \\
\hline HB $\operatorname{sam} 16 \mathrm{~F}$ & TATGACATGATTACGAATTCGAGCACCACGACTACGACACGATGAAGGCCG \\
\hline HB sam $16 \mathrm{R}$ & GCTATGACATGATTACGAATTCTCATCCGTCGGCTCGGTACTTGGCGG \\
\hline sam 17 Left F & CGGTTGGTAGGATCCACATATGCGCGTCCTCTTCACCTGCCACGC \\
\hline sam17 Left R & CGACGGCCAGTGCCAAGCTTTCGATCTGGTCGTGCACGGTGGCCG \\
\hline sam17 Right $\mathrm{F}$ & ACCGTGCACTACCACTACGACCAGCCGATCCTC \\
\hline sam17 Right $\mathrm{R}$ & TCGTAGTGGTAGTGCACGGTCTCGGCCTGCAACGCCTCCTCGGAGT \\
\hline $\mathrm{HB} \operatorname{sam} 17 \mathrm{~F}$ & TATGACATGATTACGAATTCCGAGGACATCGGCTGGGAGGAGTTCCG \\
\hline HB $\operatorname{sam} 17 \mathrm{R}$ & GCTATGACATGATTACGAATTCTCAGCGGGTGCGGAACTTGGCGGTCA \\
\hline sam18 Left F & CGGTTGGTAGGATCCACATATGCGCGTCCTGTTCGCCGCCAATCC \\
\hline sam18 Left R & GGCCAGTGCCAAGCTTTACTTGGCGGTGAGCTCTTCCAGTCGCGGGAC \\
\hline sam18 Right $\mathrm{F}$ & GAATTCCATATGCTGGTCTCCTACGTGCCGCTCAACGAGCTG \\
\hline sam18 Right $\mathrm{R}$ & CCAGCATATGGAATTCGAAGGTGGGGTGGCAGGCGAACC \\
\hline HB sam $18 \mathrm{~F}$ & ACATGATTACGAATTCTTCACCACGATCTCGAAGGTGGTCG \\
\hline HB $\operatorname{sam} 18 \mathrm{R}$ & GCTATGACATGATTACGAATTCTCAGCGGACGGCGAGTTCTTCCAGCGCCG \\
\hline sam19 Left F & CGGTTGGTAGGATCCACATATGGTGAGGTCGGTGCGAGTCCTGTTCACGA \\
\hline sam19 Left R & CGACGGCCAGTGCCAAGCTTATCGGCTGGTCGTAGTGGTAGTGCACGGTG \\
\hline sam19 Right $\mathrm{F}$ & CGGGGTGTCCGAGGAGATGATGTGGGACTTCGACGAGCCCGAACTGGCGC \\
\hline sam19 Right $\mathrm{R}$ & CATCATCTCCTCGGACACCCCGGTGTGCCGCAGCACCTGGAACAGGT \\
\hline HB sam $19 \mathrm{~F}$ & TATGACATGATTACGAATTCACGTCGGACGCTGTTCGTGCGGCCGGTGGCG \\
\hline HB sam $19 \mathrm{R}$ & GCTATGACATGATTACGAATTCTCACCCCCCGATCCGGTAGTCGGCCGTGAGC \\
\hline sam 20 Left F & CGGTTGGTAGGATCCACATATGCGTGTCCTGCTCGCCGCGAACCCGGACAAG \\
\hline sam 20 Left R & CGACGGCCAGTGCCAAGCTTAGGTACAGGAAGATCGCCTTCTCCGGATTGGC \\
\hline sam20 Right $\mathrm{F}$ & CTGCACTACCACTACGACCAGCCGATCCTGGCG \\
\hline sam20 Right $\mathrm{R}$ & TGGTCGTAGTGGTAGTGCAGCGACTCGACCTTCGCCGCGTCGCCCGCCG \\
\hline HB sam $20 \mathrm{~F}$ & TATGACATGATTACGAATTCTAATAGGTTGGGGACCCAAATGCG \\
\hline $\mathrm{HB} \operatorname{sam} 20 \mathrm{R}$ & GCTATGACATGATTACGAATTCTCAGCGGGCGCGGTGCTTCGCGGT \\
\hline
\end{tabular}


Table S3. Strains used in this study.

\begin{tabular}{|c|c|c|}
\hline Strains & Relevant comments & Source/Reference \\
\hline E. coli $\mathrm{DH} 5 \alpha$ & Conventional cloning host strain & Invitrogen \\
\hline E. coli $\mathrm{DH} 10 \mathrm{~B}$ & F-, mcrA $\Delta($ mrr-hsdRMS-mcrBC) & Invitrogen \\
\hline E. coli $\mathrm{ET} 12567 / \mathrm{pUZ} 8002$ & $\begin{array}{l}\text { DNA non methylation host, containing the } \\
\text { conjunction helper plasmid pUZ8002, } \operatorname{Kan}^{\mathrm{R}}\end{array}$ & 1 \\
\hline Saccharothrix espanaensis & Saccharomicins producing bacteria & 2 \\
\hline FDU20-11 & sam11 in-frame deletion mutant & \\
\hline FDU20-12 & sam 12 in-frame deletion mutant & \\
\hline FDU20-13 & sam13 in-frame deletion mutant & \\
\hline FDU20-14 & sam 14 in-frame deletion mutant & \\
\hline FDU20-15 & sam 15 in-frame deletion mutant & This study \\
\hline FDU20-16 & sam 16 in-frame deletion mutant & \\
\hline FDU20-17 & sam 17 in-frame deletion mutant & \\
\hline FDU20-18 & sam 18 in-frame deletion mutant & \\
\hline FDU20-19 & sam 19 in-frame deletion mutant & \\
\hline FDU20-20 & sam 20 in-frame deletion mutant & \\
\hline FDU20-11HB & sam 11 complementation mutant & \\
\hline FDU20-12HB & sam 12 complementation mutant & \\
\hline FDU20-13HB & sam 13 complementation mutant & \\
\hline FDU20-14HB & sam 14 complementation mutant & \\
\hline FDU20-15HB & sam 15 complementation mutant & This study \\
\hline FDU20-16HB & sam 16 complementation mutant & \\
\hline FDU20-17HB & sam 17 complementation mutant & \\
\hline FDU20-18HB & sam 18 complementation mutant & \\
\hline FDU20-19HB & sam 19 complementation mutant & \\
\hline FDU20-20HB & sam 20 complementation mutant & \\
\hline
\end{tabular}


Table S4. Plasmids used in this study.

\begin{tabular}{|c|c|c|}
\hline Plasmids & Relevant genotype/comments & Source \\
\hline pKC1139 & oriT, ori(psG5), aacIV(3), $6.5 \mathrm{~kb}$ & 3 \\
\hline pIB139 & $\begin{array}{l}\text { NdeI and EcoRI digestion, erythromycin resistance gene } \\
\text { containing a strong promoter of the Streptomyces PermEX } \\
\text { integrating vectors }\end{array}$ & 4 \\
\hline pFDU20-11 & sam11 in-frame deletion costruction & This study \\
\hline pFDU20-12 & sam 12 in-frame deletion costruction & This study \\
\hline pFDU20-13 & sam13 in-frame deletion costruction & This study \\
\hline pFDU20-14 & sam 14 in-frame deletion costruction & This study \\
\hline pFDU20-15 & sam 15 in-frame deletion costruction & This study \\
\hline pFDU20-16 & sam 16 in-frame deletion costruction & This study \\
\hline pFDU20-17 & sam 17 in-frame deletion costruction & This study \\
\hline pFDU20-18 & sam18 in-frame deletion costruction & This study \\
\hline pFDU20-19 & sam19 in-frame deletion costruction & This study \\
\hline pFDU20-20 & sam20 in-frame deletion costruction & This study \\
\hline pHB-sam11 & sam11 complementation & This study \\
\hline pHB-sam 12 & sam 12 complementation & This study \\
\hline pHB-sam 13 & sam13 complementation & This study \\
\hline pHB-sam14 & sam14 complementation & This study \\
\hline pHB-sam15 & sam15 complementation & This study \\
\hline pHB-sam 16 & sam16 complementation & This study \\
\hline pHB-sam17 & sam17 complementation & This study \\
\hline pHB-sam 18 & sam 18 complementation & This study \\
\hline pHB-sam 19 & sam19 complementation & This study \\
\hline pHB-sam 20 & sam 20 complementation & This study \\
\hline
\end{tabular}




\section{References}

1. Flett, F.; Mersinias, V.; Smith, C. P., High efficiency intergeneric conjugal transfer of plasmid DNA from Escherichia coli to methyl DNA-restricting streptomycetes. FEMS Microbiol Lett 1997, 155(2), 223-9.

2. Strobel, T.; Al-Dilaimi, A.; Blom, J.; Gessner, A.; Kalinowski, J.; Luzhetska, M.; Puhler, A.; Szczepanowski, R.; Bechthold, A.; Ruckert, C., Complete genome sequence of Saccharothrix espanaensis DSM 44229(T) and comparison to the other completely sequenced Pseudonocardiaceae. Bmc Genomics 2012, 13, 465.

3. Bierman, M.; Logan, R.; O'Brien, K.; Seno, E. T.; Rao, R. N.; Schoner, B. E., Plasmid cloning vectors for the conjugal transfer of DNA from Escherichia coli to Streptomyces spp. Gene 1992, 116(1), 43-9.

4. Wilkinson, C. J.; Hughes-Thomas, Z. A.; Martin, C. J.; Bohm, I.; Mironenko, T.; Deacon, M.; Wheatcroft, M.; Wirtz, G.; Staunton, J.; Leadlay, P. F., Increasing the efficiency of heterologous promoters in actinomycetes. $J$ Mol Microbiol Biotechno/ 2002, 4 (4), 417-26. 